\title{
Cloud Synergetic Recommendation Model for Overseas Chinese Education by Modeling Multi-Source User Metaphor Information
}

\author{
https://doi.org/10.3991/ijet.v14i23.11105 \\ Zhehuang Huang \\ Huaqiao University, Quanzhou, Fujian \\ startstartstart1@163.com
}

\begin{abstract}
With the deepening of global economic integration, overseas Chinese education has attracted much attention. How to retrieve resources that meet user's personalized needs has become a key issue in the recommendation model of overseas Chinese education. Thus, a cloud synergetic recommendation model for overseas Chinese education is proposed in this paper to overcome this issue. Firstly, a user vector space with fine granularity representation is constructed by introducing multi-knowledge source emotional metaphor information. Secondly, considering the synergetic interaction among various user features, a synergetic user group recognition method based on synergetic theory is proposed. Finally, in order to improve the efficiency of resource acquisition, a recommendation model of Chinese education based on cloud computing is presented.
\end{abstract}

Keywords - Online learning, overseas Chinese education, cloud computing, recommendation Model

\section{Introduction}

In recent years, with the internationalization of economic development, cultural exchanges around the world have become more and more frequent. As a result, overseas Chinese education has been gained increased attention [1,2]. With the development of mobile internet, intelligent terminals and cloud computing technology, various educational resources have shown explosive growth, data density has increased unprecedentedly, and the big data era is gradually unfolding. At present, big data technology has been widely used in E-learning [3-6], X-Learning [7] and M-learning [8]. In the big data age, how to make full use of big data technology to obtain resources that meet the personalized needs of users has become a major task of recommendation model for overseas Chinese education.

As the basis and core of personalized information service, user model is directly related to the quality of Chinese education recommendation model. Only when user's personal interests, domain preferences and browsing history are fully understood, can we share resources according to the user's characteristics, and then realize the ideal 
personalized service. In order to improve the precision of user description, it is necessary to add multi-knowledge source information into user model. At the same time, messages and critical information of users often imply a large number of metaphors. Only analyzing and processing the metaphors and emotional information implied in these texts quickly, can we better meet the needs of user modeling.

On the other hand, how to embody the interdependence and mutual restriction among the various characteristic information of user is a key problem. Synergetics is a comprehensive discipline which studies the evolution of synergetic system from disorder to order. Synergetic pattern recognition has been successfully used in semantic annotation [9], automatic control [10] and semantic role labeling [11]. One of the advantages of synergetic processing method is its strong anti-defect ability. Using this method to realize user group identification can better represent the global constraints of user group information, and then can better deal with the matching problem of incomplete user information.

Another key issue in building synergetic recommendation model is the implementation of real-time sharing. Synergetic user identification can achieve better recognition performance, but the relatively large amount of computation limits its real-time application. How to improve the recognition efficiency is an urgent problem to be solved. Cloud computing [12,13] technology provides a network application development model. It studies how to divide a large amount of computing tasks into many small parts, then distribute these parts to the computer on the network for distributed processing, and finally merge the returned contents to get the final results [14]. There is data parallelism in the process of user group analysis, so the identification time can be shortened by parallel computing to achieve the purpose of real-time resource sharing. Thus, this paper proposes a cloud synergetic recommendation model for overseas Chinese education.

\section{User modeling with multi-knowledge source emotional metaphor information}

The construction and updating of user model is an important issue in the Chinese education model. Vector space model is used to express user's personalized information for its computability and operability. There are some shortcomings in the traditional user vector space, which are mainly reflected in two aspects: (i) the granularity of user's personalized information expression is not fine enough, the knowledge source is relatively single, and the information is not rich enough. (ii) The understanding of emotional and metaphorical information in various sources of knowledge is not fully considered.

Therefore, the above shortcomings of traditional vector space are improved in this section. Firstly, a fine-grained vector semantic space from multiple is constructed. knowledge sources such as domain interests, personal preferences and browsing history. Secondly, implicit emotional and metaphorical information can be obtained from multiple knowledge sources. The improved user model is shown in Figure 1. 


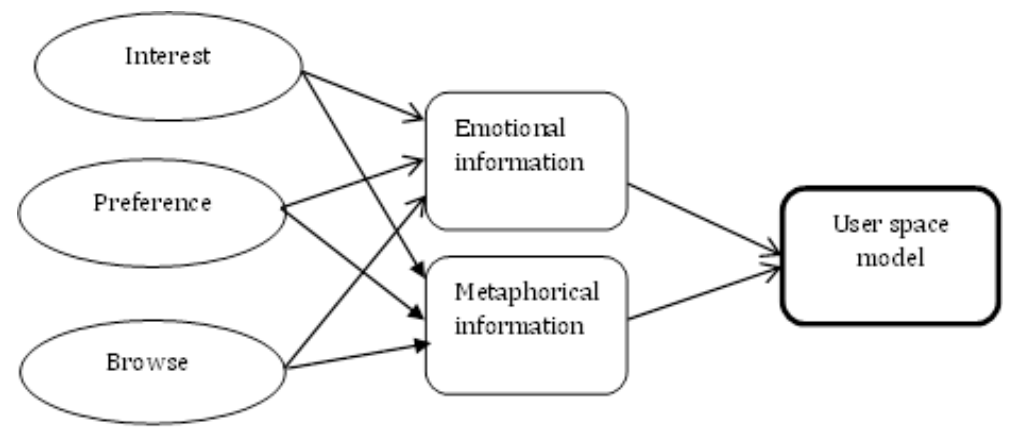

Fig. 1. User Modeling

\section{Synergetic User Identification Model}

A key problem of synergetic recommendation model for Chinese education is user group identification. User group identification is a process in which each feature interacts and restricts each other. The recognition process can be regarded as a competition process of many order parameters. A certain number of order parameters are constructed according to the test mode $u$ and the prototype mode, and the dynamic equation is used to evolve to complete the identification of user $u$, as shown in Figure 2.

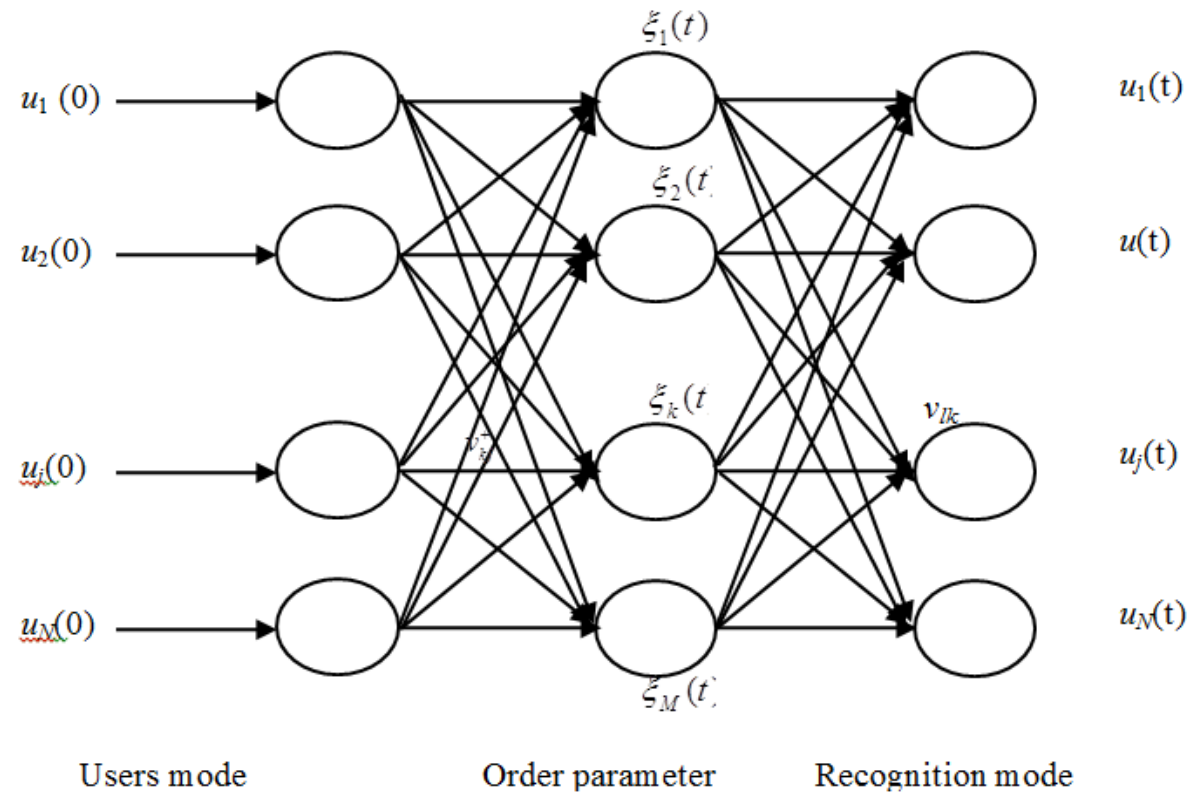

Fig. 2. Synergetic recommendation model 
Order parameter in intermediate layer:

$$
\xi_{k}(0)=\sum_{j=1}^{N} v_{k j}^{+} u_{j}(0)
$$

The output mode is:

$$
u_{l}(t)=\sum_{k=1}^{M} \xi_{k}(t) v_{l k}, l=1,2, \mathrm{~L}, N
$$

In order to reflect the mutual guidance and driving relationship among different knowledge sources, such as interest, preference and emotion of different users of Chinese education, the reconstructed evolution equation is reconstructed to make effective use of prior knowledge. In this way, only one order parameter wins at the order parameter layer.

The dynamic equation of the order parameter is as follows: $\xi_{\mathrm{k}}(\mathrm{n}+1)-\xi_{\mathrm{k}}(\mathrm{n})=r\left(\lambda_{k}-D+B \xi_{k}^{2}(\mathrm{n})\right) \xi_{\mathrm{k}}(\mathrm{n})$

,Where

$$
\mathrm{D}=(\mathrm{B}+\mathrm{C}) \sum_{\mathrm{k}^{\prime}} \xi_{\mathrm{k}^{\prime}}^{2}(\mathrm{n})
$$

$n$ is the current number of iterations.

In the dynamic evolution equation, attention parameter $\lambda_{k}$ has an important influence on the identification performance of the model. Different settings will lead to different recognition results. How to reconstruct the attention parameters to reflect the interaction among the factors in the user vector model is a key problem. Based on the constraints of user vectors, the attention parameters are reconstructed.

Assume that $u$ is a user and $g_{j}(u),(j=1,2, \mathrm{~L}, m)$ is the characteristic function of $u$. It can be defined as:

$$
\lambda_{k}(u)=\sum_{j} \gamma_{j} g_{j}(u),(j=1,2, \mathrm{~L}, m)
$$

Where $\gamma_{j}$ is the weight of characteristic function, and the $g_{j}(u),(j=1,2, \mathrm{~L}, m)$ can be constructed from constraints among users' multiknowledge source information。

The implementation of user synergetic identification model includes the following steps:

- Modeling user space vectors from multiple knowledge sources; 
- Constructing order parameters which can reflect the similarity between prototype mode and test mode;

- Reconstructing the evolution equation of ordinal parameters;

- Setting network parameters;

- Recognition modes are obtained through the evolution of the order parameter equation.

\section{$4 \quad$ Synergetic Recommendation Model Based on Cloud Computing}

Hadoop $[15,16]$ is a typical cloud computing platform, which can realize distributed computing of massive data in a cluster composed of a large number of computers. Hadoop distributes data among available computer clusters and completes computational tasks. These clusters can be easily extended to thousands of nodes. Map-Reduce is an implementation mechanism of Hadoop, which is responsible for computing large amounts of data [17]. The basic parallel computing tasks can be realized by using Map and Reduce functions. These two functions provide abstract operation and parallel programming interface to complete large-scale data processing simply and conveniently.

The synergetic recommendation model based on cloud computing is shown in Figure 3. The model consists of cloud monitor, users pool and cloud server pool.

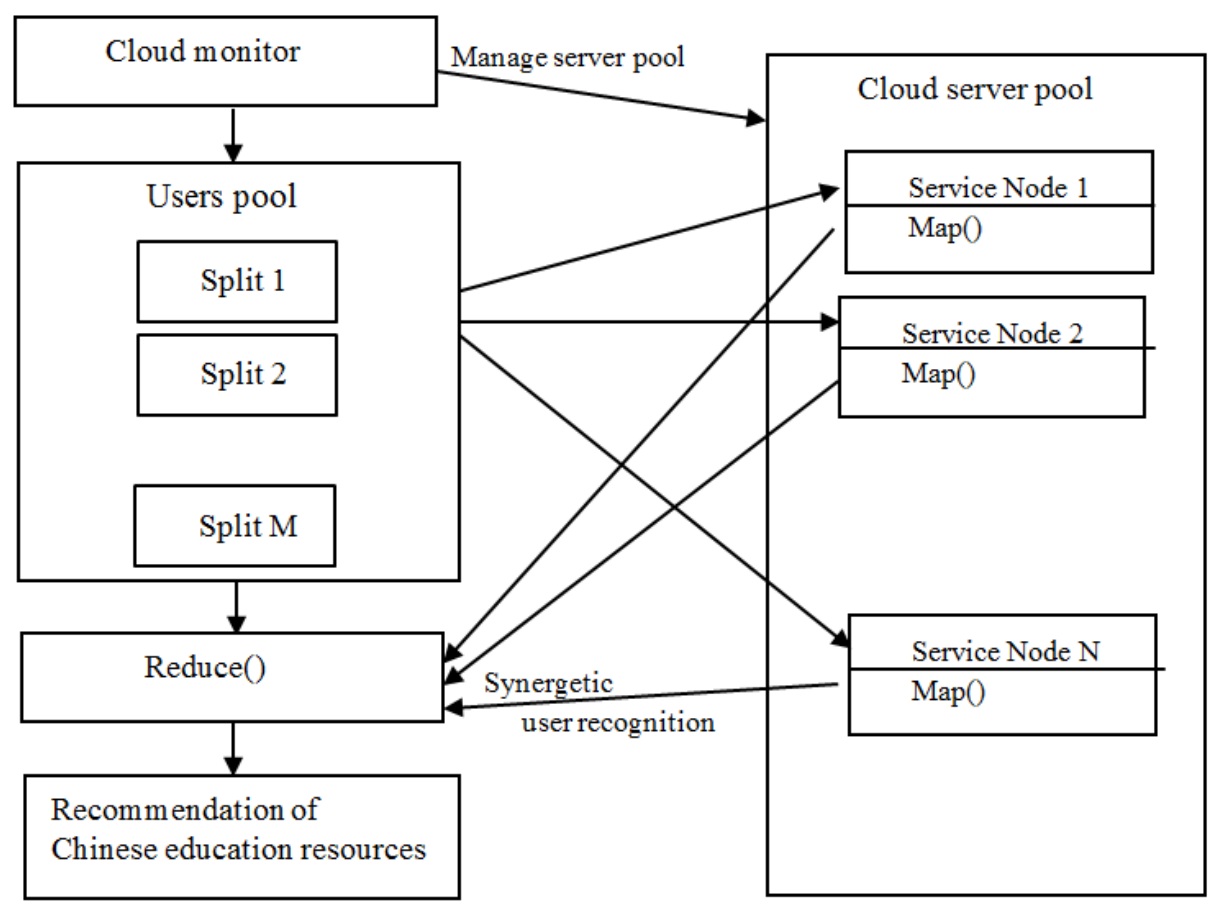


Fig. 3. Synergetic User Recommendation Model Based on Cloud Computing

The implementation of recommendation model based on Map-Reduce mechanism includes the following steps:

- Constructing user vector space with fine granularity representation from multiple knowledge sources such as domain interests, personal preferences and browsing history

- Obtain implicit emotional and metaphorical information from multiple sources

- of knowledge

- User partitioning information is sent to each service node for user identification. Each service node completes the following steps

○ Map function completes the synergetic recognition of user modes through the evolution of order parameters.

○ Return the identification result to cloud monitor.

- Merge the identification results in Reduce stage.

- Recommend Chinese education resources based on user's personalized information.

\section{Experimental simulation}

In order to better illustrate the user's synergetic identification process, an example is given below. Assuming that the user has five candidate modes, the order parameters and parameters $\lambda_{k}$ are set as shown in Table 1 .

Table 1. Order parameters and attention parameters of candidate user modes

\begin{tabular}{|l|c|c|}
\hline \multicolumn{1}{|c|}{$\begin{array}{c}\text { Candidate } \\
\text { user modes }\end{array}$} & $\begin{array}{c}\text { Order parameter } \\
\text { of User modes }\end{array}$ & $\lambda_{k}$ \\
\hline User mode 1 & 0.244 & 0.488 \\
User mode 2 & 0.216 & 0.648 \\
User mode 3 & 0.182 & 0.182 \\
User mode 4 & 0.181 & 0.362 \\
User mode 5 & 0.177 & 0.354 \\
\hline
\end{tabular}

Thus, the best user mode can be obtained by the evolution of order parameter equation. As seen in Figure 4, user mode 2 has won through the evolution and competition of order parameters. 


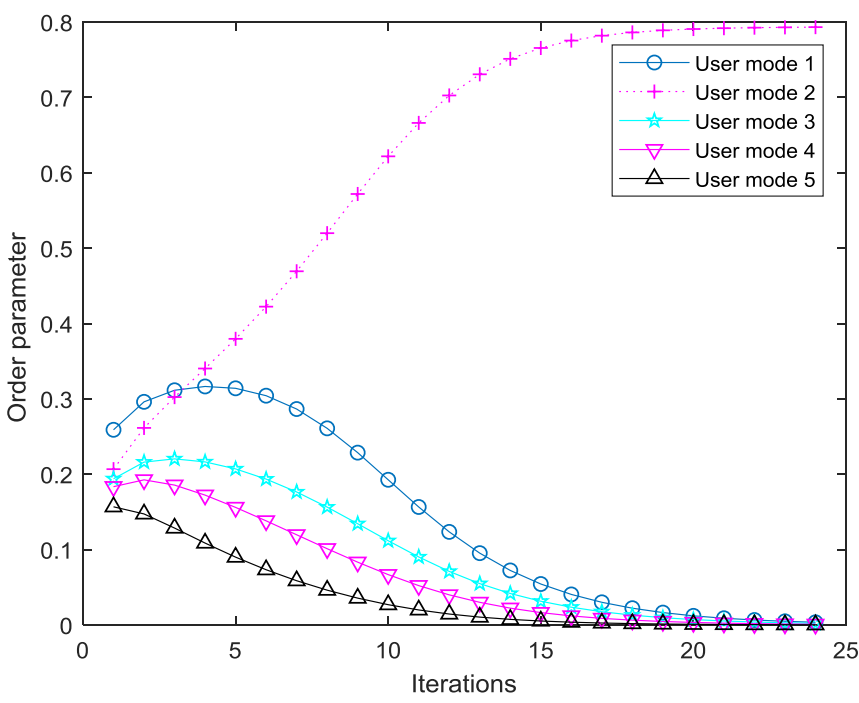

Fig. 4. Evolution process of users order parameters

After identifying the user mode, Chinese education resources that meet individual needs can be recommended according to user mode.

\section{Conclusion}

In this paper, a synergetic recommendation model is implemented based on cloud computing. The following conclusions can be drawn

- By introducing emotional and metaphorical information from multiple knowledge sources, user modes can be better modeled

- User modes identification process can be regarded as a competitive process of various features. Through the evolution of order parameters, user modes can be identified

- Map-Reduce model can improve the efficiency of synergetic user recommendation model

Some problems related to synergetic recommendation model need to be discussed further. They are summarized as follows. First, logical constraints in user's various features play an important role in synergetic recognition, and more possible constraints need to be further obtained. Second, reconstruction of evolutionary equation may have better recognition effect, so it is necessary to study it in depth. 


\section{$7 \quad$ References}

[1] D Starr. "Chinese Language Education in Europe: the Confucius Institutes". European Journal of Education,vol 44, no 1, 2009, pp.65-82. https://doi.org/10.1111/j.1465-3435.20 $\underline{08.01371 . x}$

[2] Yang R. "Soft Power and Higher Education: An Examination of China's Confucius Institutes Globalisation", Societies and Education,vol 8, no 2, 2010, pp.235-245. https://doi. org/10.1080/14767721003779746

[3] Gusev M, Ristov S, Velkoski G, et al. "E-learning and benchmarking platform for parallel and distributed computing". International Journal of Emerging Technologies in Learning, vol.9, no.2,2014,pp.17-21. https://doi.org/10.3991/ijet.v9i2.3215

[4] Mhouti A E, Erradi M, Nasseh A. "Using cloud computing services in e-learning process: Benefits and challenges". Education \& Information Technologies,vol.23,no 2,2018,pp.893909. https://doi.org/10.1007/s10639-017-9642-x.

[5] Tarus J K, Niu Z, Yousif A. "A hybrid knowledge-based recommender system for elearning based on ontology and sequential pattern mining". Future Generation Computer Systems, vol.72,2017, pp.37-48. https://doi.org/10.1016/j.future.2017.02.049

[6] Henda Chorfi . "Optimizing E-Learning Cognitive Ergonomics Based on Structural Analysis of Dynamic Responses", International Journal of Emerging Technologies in Learning,vol.14,no.10, 2019,pp. 132-149. https://doi.org/10.3991/ijet.v14i10.10134

[7] Xiangsheng K. "Big Data X-Learning Resources Integration and Processing in Cloud Environments". International Journal of Emerging Technologies in Learning, vol.9, no.5, 2014, pp.22-26. https://doi.org/10.3991/ijet.v9i5.3783

[8] Joo-Nagata J, Martinez Abad F, Garca-Bermejo Giner J, et al. "Augmented reality and pedestrian navigation through its implementation in m-learning and e-learning". Computers \& Education, vol.111,2017, pp.1-17. https://doi.org/10.1016/j.compedu.2017.04.003

[9] Huang Z, Chen Y. "An Integration Model of Semantic Annotation Based on Synergetic Neural Network". Intelligent Automation \& Soft Computing, vol.22,no.3,2016,pp.525532. https://doi.org/10.1080/10798587.2016.1158498

[10] Wang W Z, Pan L and Liu B H, "Synergetic method of traffic state recognition based on manifold learning", in: Proceedings of the IEEE International Conference on Automation and Logistics, Shenyang, China 2009,pp. 587-591. https://doi.org/10.1109/ical.2009.52628 $\underline{56}$

[11] Chen Y, Huang Z, Shi X. "An SNN-Based Semantic Role Labeling Model with Its Network Parameters Optimized Using an Improved PSO Algorithm". Neural Processing Letters,vol.44,n0.1, 2016,pp.245-263. https://doi.org/10.1007/s11063-015-9449-y

[12] Varghese B, Buyya R. "Next generation cloud computing: New trends and research directions". Future Generation Computer Systems,vol.79,2018,pp.849-861. https://doi.org/10.10 16/j.future.2017.09.020

[13] Yang C, Huang Q, Li Z, et al. "Big Data and cloud computing: innovation opportunities and challenges". International Journal of Digital Earth,vol.10,no.1, 2017,pp.13-53. https:// doi.org/10.1080/17538947.2016.1239771

[14] Aldhuraibi Y, Paraiso F, Djarallah N, et al. "Elasticity in Cloud Computing: State of the Art and Research Challenges". IEEE Transactions on Services Computing,vol.11,no.2, 2018,pp.430-447. https://doi.org/10.1109/tsc.2017.2711009

[15] Whaiduzzaman M, Sookhak M, Gani A, et al. "A survey on vehicular cloud computing". Journal of Network and Computer Applications, vol.40,2014, pp.325-344. https://doi.org/ $\underline{10.1016 / j . j n c a .2013 .08 .004}$ 
[16] Abdel-Basset M, Mai M, Chang V. “NMCDA: A framework for evaluating cloud computing services”. Future Generation Computer Systems,vol.86, 2018,12-29. https://doi.org/ 10.1016/j.future.2018.03.014

[17] Jian S, Zhou T, Chen X, et al. "Anonymous and Traceable Group Data Sharing in Cloud Computing". IEEE Transactions on Information Forensics \& Security, vol.13,no.4,2018,pp.912-925. https://doi.org/10.1109/tifs.2017.2774439

\section{Author}

Zhehuang Huang: Corresponding author. He is an associate professor in the School of Mathematics Sciences, Huaqiao University, 362021, Quanzhou, China. His research interest covers E-learning, machine learning, intelligent algorithm and data fusion.

This work was supported by the High-level Talents Start-up Project of Huaqiao University (16BS814) and the Natural Science Foundation of Fujian Province (2017J01114).

Article submitted 2019-06-24. Resubmitted 2019-08-13. Final acceptance 2019-08-29. Final version published as submitted by the authors. 